\title{
Lamtobathynella pentodonta n.g., n.sp., Leptobathynellidé nouveau d'Afrique (Côte d'Ivoire) (Malacostraca, Bathynellacea)
}

\author{
Eugène Serban* et Nicole Coineau**
}

\author{
SUMMARY
}

Lamtobathynella pentodonta n.g., n.sp. is described. We consider then its relations with the genus Leptobathynella and Parvulobathynella. Some observations about the most characteristic features of the VIIIth male pereiopods are made. This study ends with a discussion concerning the differences between Lamtobathynella pentodonta n.g., n.sp. and Acanthobathynella knoepffleri Coineau.

\section{INTRODUCTION}

L'étude des récoltes de faune interstitielle provenant de Côte d'Ivoire a donné lieu à la description des genres Acanthobathynella Coineau et Haplophallonella Serban et Coineau'.

Acanthobathynella knoepffleri Coineau (Coineau, 1967), premier représentant de l'ordre des Bathynellacea trouvé dans l'ouest africain et le premier Leptobathynellidé découvert en Afrique, demeure, 15 ans après sa publication, une forme singulière. Il s'individualise par deux caractéristiques principales: la mandibule comporte une partie masticatrice très simplifiée et la chétotaxie générale se compose de courtes soies à l'aspect d'épines. La première caractéristique éloigne nettement cette espèce des représentants des autres genres de la famille des

1) Prélèvements effectués en 1969 par L. - P. Knoepffler, que nous remercions.

(*) Institut de Spéologie «Emile Racovitza», 9 rue Mihail Moxa, Bucarest, Roumanie.

(**) Laboratoire Arago, 66650 Banyuls-sur-Mer, France. 
Leptobatynellidae (Brasilibathynella Jakobi, Leptobathynella Noodt, Parvulobathynella Schminke, Odontobathynella Delamare et Serban), tandis que la seconde est inédite dans l'ordre des Bathynellacea.

Haplophalonella heterodonta Serban et Coineau (Serban et Coineau, 1975), le premier Parabathynellidé decrit de Côte d'Ivoire, présente des affinités avec les genres Cteniobathynella Schminke, Habrobathynella Schminke et Ctenophallonella Coineau et Serban. A la suite de l'étude de ce représentant, nous avons reunis les quatre genres dans la tribu des Cteniobathnellini. Ce point de vue diverge de celui de Schminke (1973) qui considère que la famille des Leptobathynellidae Noodt, est synonyme de la famille des Parabathynellidae Noodt, les genres Brasilibathynella, Leptobathynella, Parvulobathynella et Acanthobathynella devant être réunis aux genres Cteniobathynella, Habrobathynella, Heterodontobathynella et Thermobathynella dans le «Cteniobathynella-groupe».

Outre $A$. knoepffleri et $H$. heterodonta, le matériel de Côte d'Ivoire contenait un autre représentant de la famille des Leptobahynellidés. Il s'agit d'une forme rappelant Leptobathynella et Parvulobathynella, forme que nous considérons comme espèce nouvelle, type d'un genre nouveau.

\section{DESCRIPTION}

Genre Lamtobathynella nov.

\section{Diagnose.}

Péréiopode VIII mâle: basipodite complètement englobé dans la région basale de l'appendice - région basipodiale; région pénienne glabre, comportant un grand lobe qui porte un lobule postéro-externe; lobe externe, triangulaire, bien individualisé; l'exopodite, plus long que la moitié de la largeur de la région basale du pénis, courbé, aplati dorso-ventralement, s'attache sur la face distale de la région basipodiale. Métastome à 2 dents. Mandibule à 5 dents incisives et 5 griffes sur le bord ventromédial du corps mandibulaire. La maxillule porte un endite muni de 4 phanères sur l'article proximal et 5 dents internes et 2 poils externes sur l'article distal. Article proximal de la maxille glabre. Endopodite des uropodes avec une seule griffe. Furca à 5 phanères. Antennule de 6 articles. Antenne pentaarticulée.

Lamtobathynella pentodonta n.sp.

Matériel.

1 mâle et 2 femelles provenant du milieu interstitiel du Nzi, 
situé entre Ndouci e Lamto, Côte d'Ivoire, leg. L.-Ph. Knoepffler, 6-IX-1969.

\section{Description.}

Taille: $0,65 \mathrm{~mm}-0,80 \mathrm{~mm}$.

Antennule (fig. 1A, B). Elle est formée de 6 articles. Le pédoncule, massif, est une fois et demie plus long que l'exopodite. Endopodite bien individualisé. L'article basal de l'appendice, 2 fois plus long que le $3 \mathrm{e}$ et le $6 \mathrm{e}$, pris séparément, dépasse 2,5 fois la longueur de l'article 5; les articles 2 et 4 , de même longueur, atteignent les $2 / 3$ de la longueur de l'article basal. On distingue respectivement $4,1,1,3$, 3 , et 4 poils de type a sur les articles $1,2,3$, endopodite, 5 et 6 , un poil en lancette sur chacun des articles du pédoncule et 3 sur l'article 4 . Un bâtonnet hyalin est présent sur l'article 5 et 3 sur le 6 e.

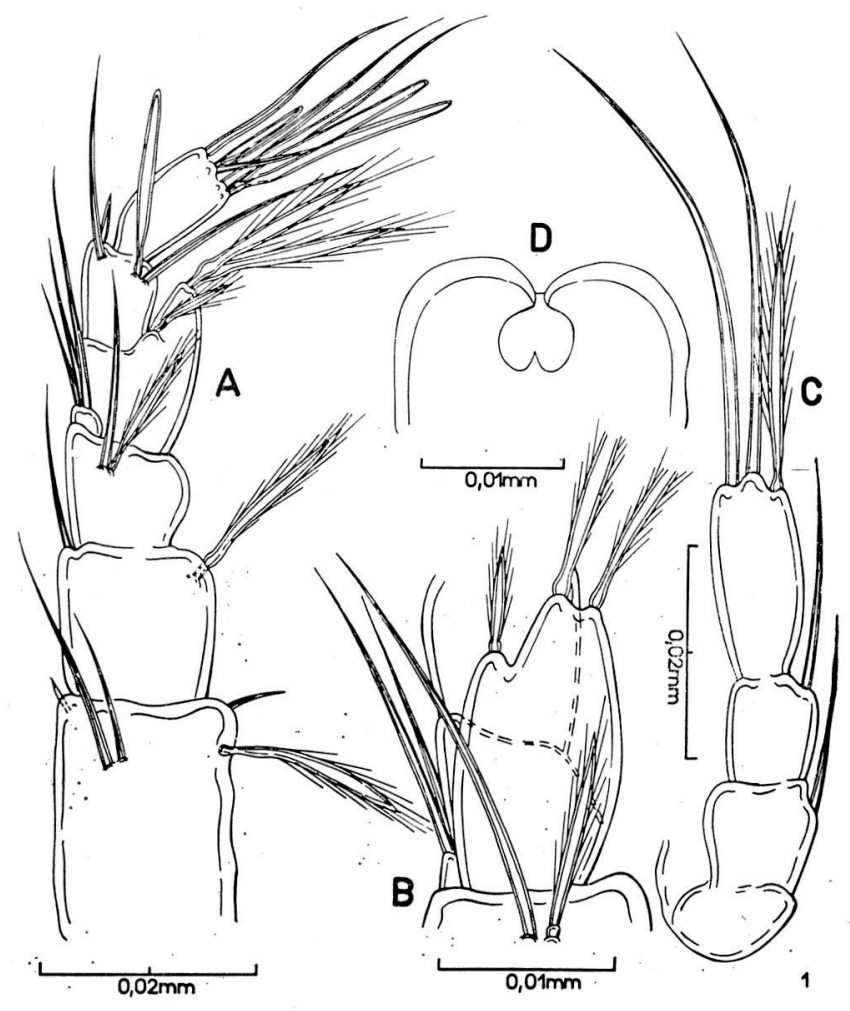

Fig. 1 - Lamtobathynella pentodonta n. g., n. sp. A, antennule; B, article 4 antennulaire (détail); C, antenne; D, labrum, vue ventrale. 
Antenne (fig. 1 C). Pentaarticulée. Elle est plus courte que l'antennule de $25 \%$. Chétotaxie comprenant 5 poils: 1 sur les articles 3 et 4 et 3 sur le 5 e. Le seul poil en lancette se trouve sur l'article apical.

Labrum (figs. $1 \mathrm{D}$ et 3 ). Le contour de sa face ventrale est représenté sur la figure $1 \mathrm{D}$. Son bord est muni de soies.

Métastome (fig. 3). Cette formation porte 2 dents.

Mandibule (figs. 2 A, B et 3). D'une constitution générale semblable à celle des genres Leptobathynella et Parvulobathynella, elle porte 5 dents incisives et 5 courtes griffes; ces dernières sont situées sur le bord ventro-médial du corps mandibulaire; les 2 griffes postérieures sur un petit lobe.

Maxillule (figs. 2C et 3). L'article basal est muni d'un endite bien individualisé qui porte 4 phanères; l'article apical est pourvu de 5 dents et 2 poils.

Maxille (figs. 2D et 3). Biarticulée. L'article proximal glabre.

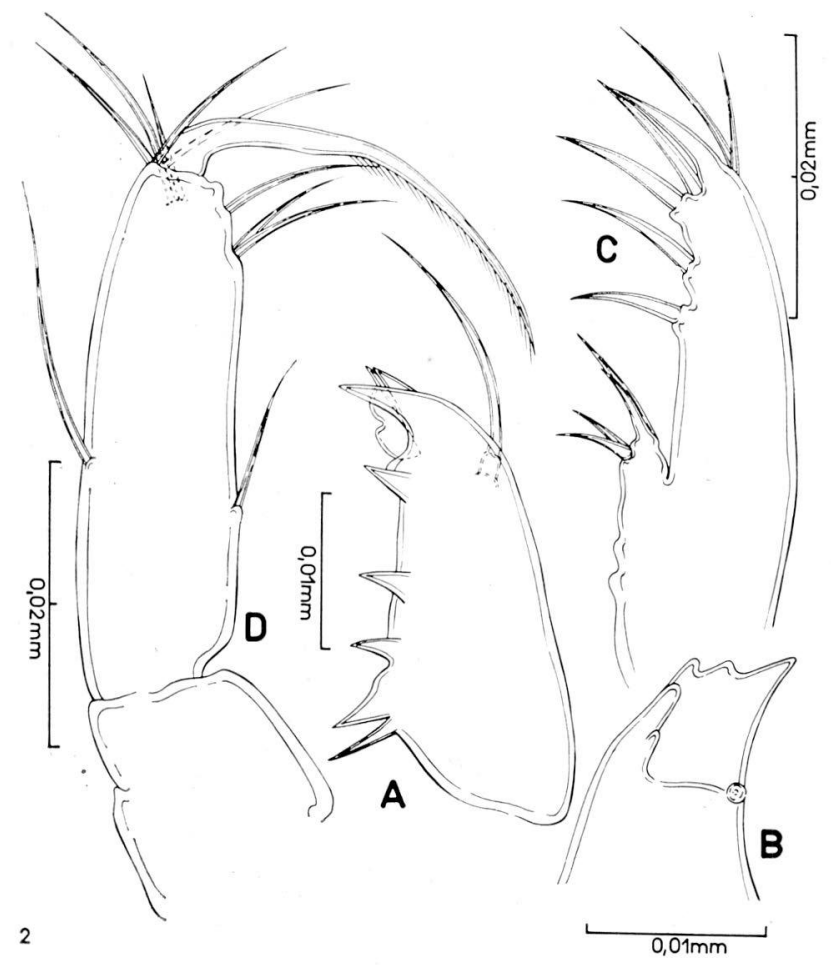

Fig. 2 - Lamtobathynella pentodonta n. g., n. sp. A, mandibule, vue ventrale; $\mathrm{B}$, partie incisive de la mandibule (détail); C, maxillule; D, maxille. 
Article distal à 10 soies et une longue griffe.

Péréiopodes I-VII (fig. 4). Les deux premières paires sans épipodites respiratoires. A partir des péréiopodes II, l'exopodite est plus long que l'endopodite et porte 2 poils apicaux et un poil situé dans la partie médiane de son bord ventral. Le poil dorsal de l'article 2, de même que les 2 phanères apicaux, sont toujours présents sur l'endopodite. Notons l'absence de cténidies sur le protopodite et sur les articles 1 et 4 de l'endopodite.

Péréiopode VIII mâle (fig. 5). Il a une longueur d'environ $0,015 \mathrm{~mm}$ et une largeur de 0,009 $\mathrm{mm}$. La région basale (Rg-bsl), ayant un contour circulaire en section transversale, occupe les

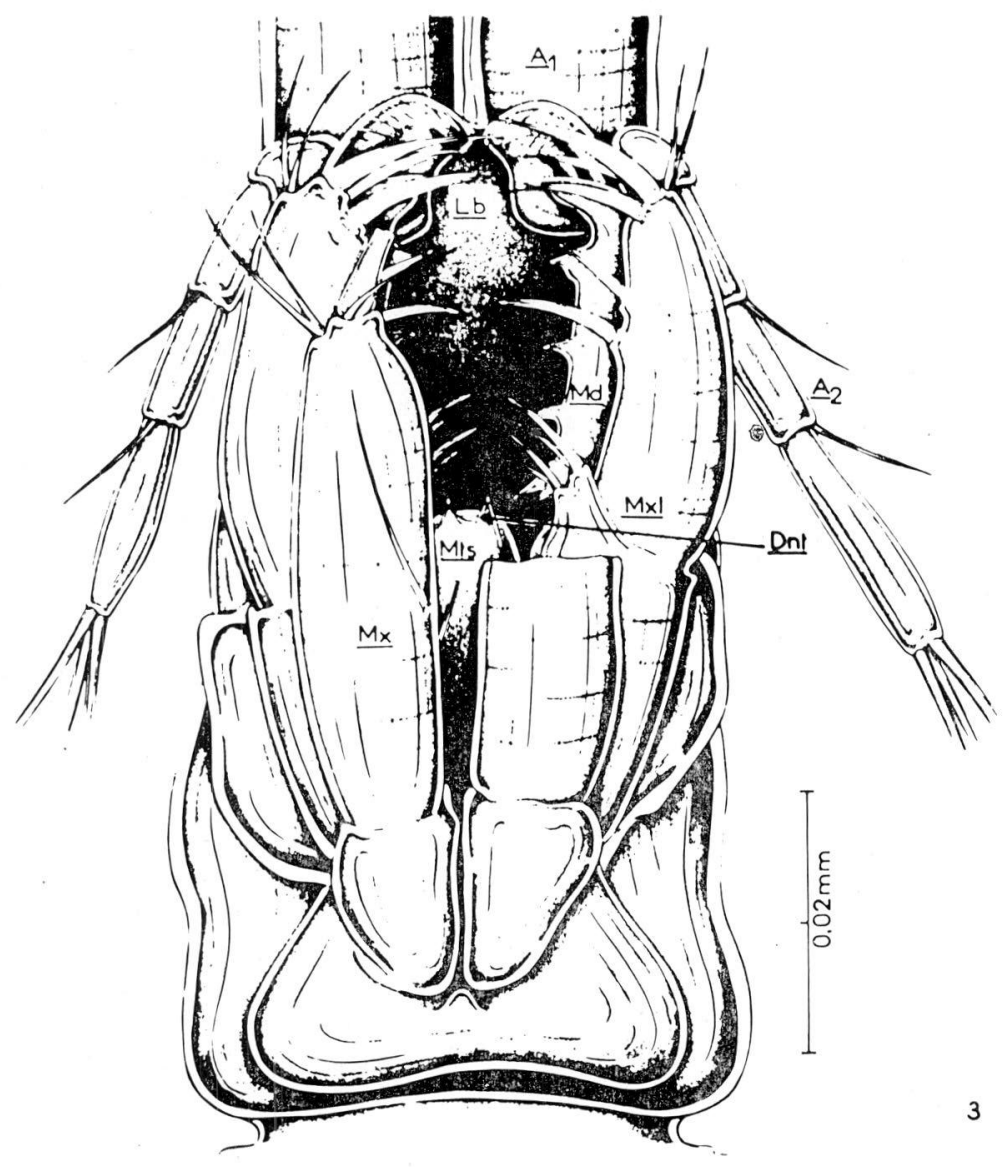

Fig. 3 - Lamtobathynella pentodonta n. g., n. sp. céphalon, vue ventrale. $\mathrm{A}_{1}$, antennule; $\mathrm{A}_{2}$, antenne; Dnt, dents du métastome; Lb, labrum; Md, mandibule; Mx, maxille; Mxl, maxillule; Mts, métastome. 
2/3 proximaux de la morphologie pénienne. La région pénienne (Rg-pn) a l'aspect d'un prolongement ventro-antérieur, 2 fois plus court que la région basale et dont l'extremité, arrondie, est la partie pénienne la plus proéminente vers le côté ventral (fig. $5 \mathrm{~B}, \mathrm{D})$; cette région est constituée par un grand lobe qui porte dans sa partie postéro-basale et externe un lobule. Nous n'avons pas observé de dents, épines ou autres formations cuticulaires ni sur le lobe, ni sur le lobule. Devant l'impossibilité à discerner les limites entre le basipodite et la région basale du pénis, nous dénommons la zone qui correspond à cet article la région basipodiale (Rg-bsp). Le lobe externe (Lb-ext), triangulaire, s'individualise au niveau de la région distale et externe de la partie basipodiale (fig. 5 B). L'exopodite (Exp.), unique rame de l'appendice, est situé sur la face distale de la région basipodiale; sa

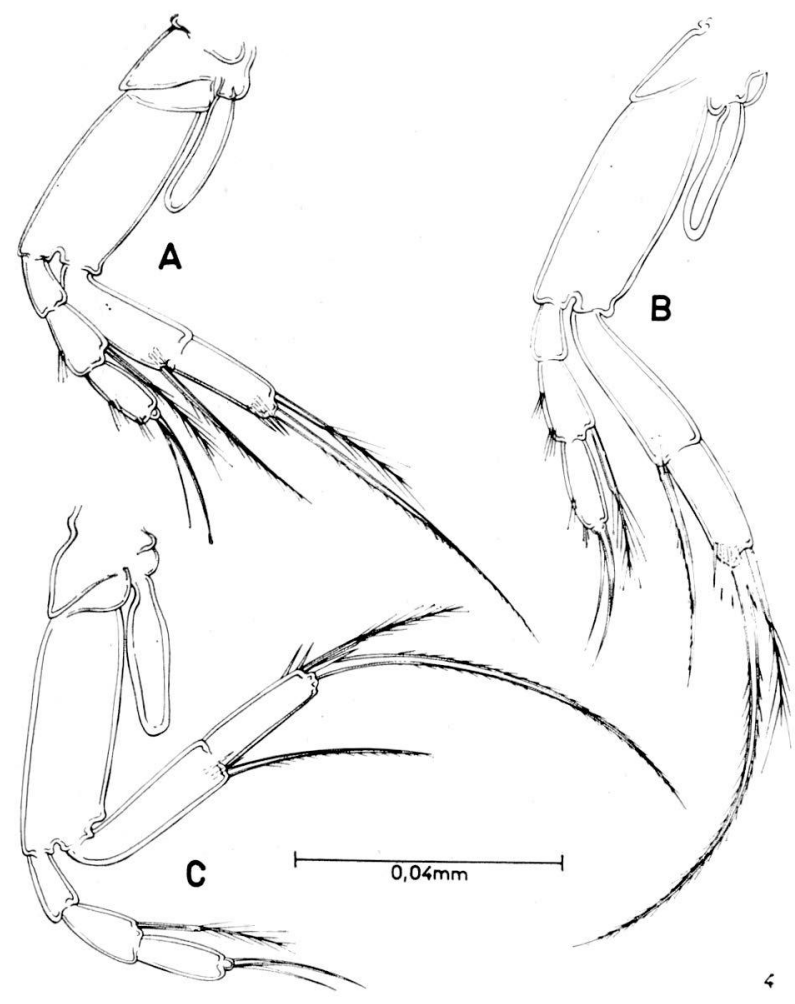

Fig. 4 - Lamtobathynella pentodonta n. g., n. sp. Péréiopodes ambulatoires. A, péréiopode $3 ; B$, péréiopode 5 ; $C$, péréiopode 7 , 
base est enfouie dans la partie caudale de la région mentionnée et l'extrémité distale orientée vers le côté rostral de l'appendice. Notons la longueur accentuée de cette rame, la forme arquée de ses faces latérales, ainsi que la forte largeur de ses faces dorsale et ventrale. La chétotaxie du péréiopode VIII mâle est représentée par l'unique poil qui se trouve dans l'angle interne et postérieur de la région basipodiale; nous pensons qu'il appartient à cette dernière, l'endopodite étant complètement réduit.

Péréiopode VIII femelle (fig. 6 A) très petit.

Uropode (fig. $6 \mathrm{~B}, \mathrm{C}$ ). Le sympodite allongé porte 2 épines. L'endopodite, un peu plus long que l'exopodite, est muni d'une longue griffe, de 2 poils apicaux et d'un poil se trouvant sur le 2e $1 / 3$ de sa longueur. L'exopodite possède 2 poils apicaux, l'un environ 3 fois plus long que la rame et l'autre très court.

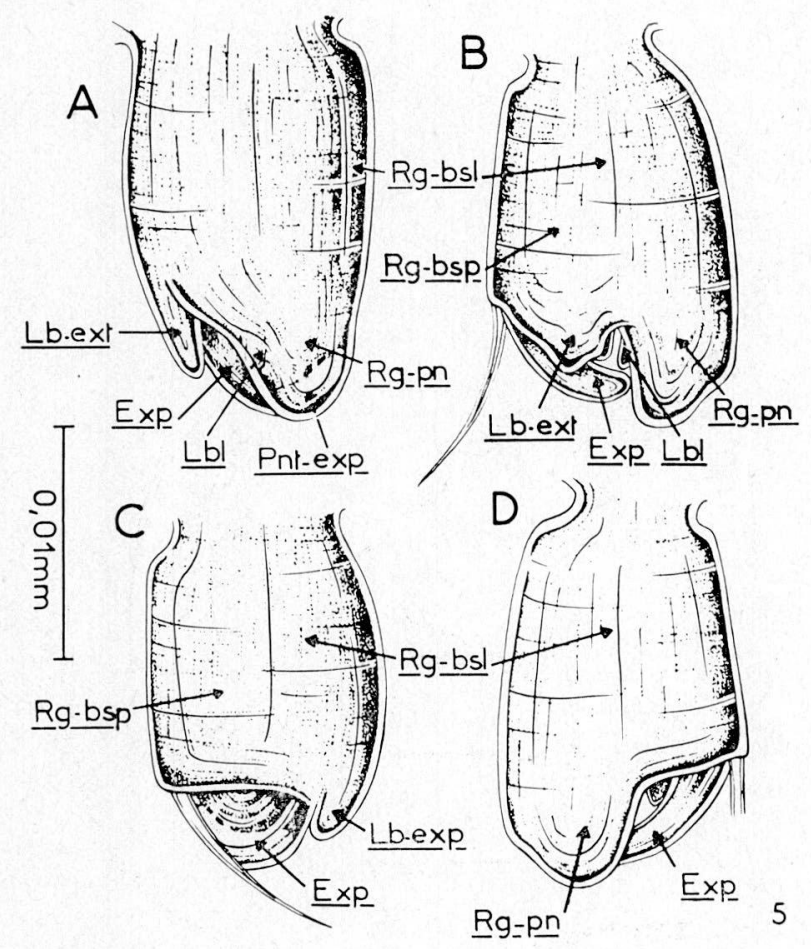

Fig. 5 - Lamtobathynella pentodonta n. g., n. sp. Péréiopode VIII mâle. A, face antérieure; B, face latéro-externe; C, face postérieure; D, face latéro-interne. Exp, exopodite; Lb-ext, lobe externe; Lbl, lobule; Pntexp, pointe exopodiale; $\mathrm{Rg}$-pn, région pénienne. 
Furca (fig. 6 B, D). Elle est pourvue de 3 griffes, d'une longue cténidie et de 2 soies.

Telson (fig. 6 B). Il est glabre et dépourvu d'opercule anal.

\section{DISCUSSION}

Les genres Leptobathynella Noodt, Parvulobathynella Schminke et Lamtobathynella n.g.

L'ensemble des caractères et, tout spécialement, la mandibule montrent que le nouveau genre présente des affinités évidentes avec Leptobathynella et Parvulobathynella dont les 6 taxa ( $L$. richerti Noodt, L. richerti brasiliensis Noodt, L. camposicola (Jakobi), Pv. ypacariensis (Noodt), Pv. camposicola (Jakobi), $P v$. riegelorum (Noodt), sont connus d'Amérique du Sud (Brésil, Argentine, Paraguay et Chili). Parmi les caractères inclus dans les diagnoses des deux genres, deux seulement apparais-

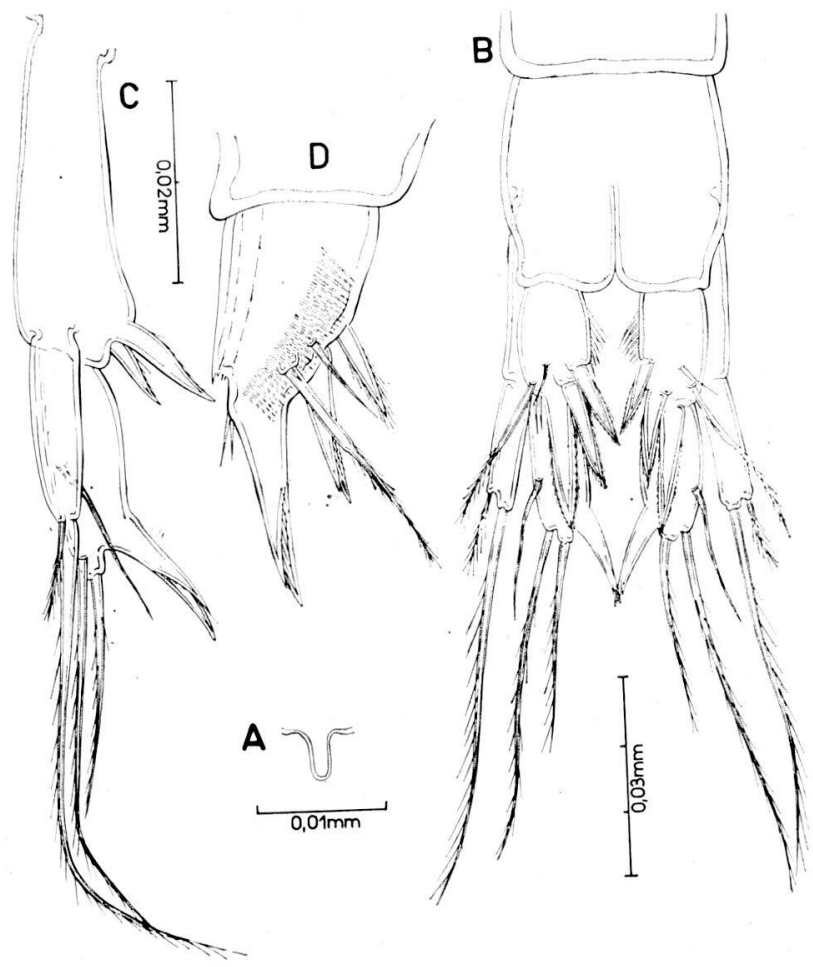

Fig. 6 - Lamtobathynella pentodonta n. g., n. sp. A, péréiopode VIII femelle; $B$, pléotelson, vue dorsale; $C$, uropode; $D$, furca, 
sent nettement distinctifs, la maxillule et la maxille, les autres étant soit identiques (antennule, antenne, mandibule, poils telsonaux), soit définis d'une manière peu concluante (endopodite uropodial, furca). Quant aux péréiopodes VIII mâles, ils ne sont pas décrits.

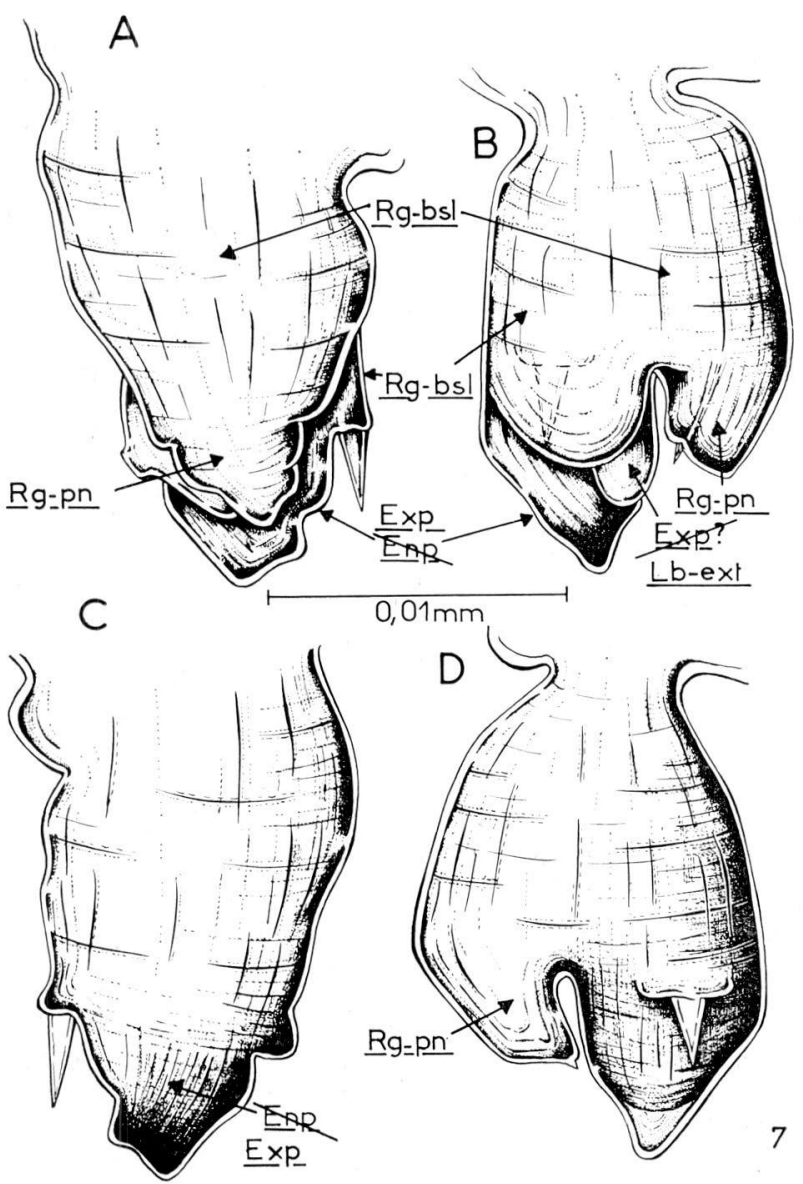

Fig. 7 - Acanthobathynella knoepffleri Coineau. Péréiopode VIII mâle. A, face antérieure; B, face latéro-externe; $C$, face postérieure; D, face latéro-interne. Exp, exopodite; Lb-ext, lobe externe; Rg-bsl, région basale; $R g-b s p$, région basipodiale; $R g$-pn, région pénienne (d'après Coineau et Serban, 1974). 
La maxillule est très différenciée chez les deux genres; il s'agit du nombre de soies, de l'absence (Leptobathynella) ou de la présence (Parvulobathynella) d'un endite sur l'article proximal et du développement (Leptobathynella) ou du non développement (Parvulobathynella) d'une crête saillante sur l'article distal. En ce qui concerne la maxille, ou bien elle porte une soie sur l'article basal (Parvulobathynella), ou bien cet article est glabre (Leptobathynella). Si l'on tient compte de cette paire de caractères, l'espèce pentodonta est intermédiaire entre les deux genres, avec une maxillule identique à celle des Parvulobathynella et une maxille identique à celle des Leptobathynella.

Il faut également noter que la mandibule de Lamtobathynella pentodonta n.g., n.sp. est la plus richement armée dans la famille des Leptobathynellidae, présentant 5 dents incisives et 5 éléments masticatoires qui sont situés sur le bord ventro-médial du corps mandibulaire.

Enfin, un autre trait qui individualise Lamtobathynella n.g. par rapport aux genres Leptobathynella et Parvulobathynella est le métastome dont le bord ventral porte 2 dents spiniformes ayant une position qui rappelle celle des paragnathes des Bathynellidés.

Le péréiopode VIII mâle de Lamtobathynella pentodonta ng., n. sp.

Il présente des traits qui se rencontrent chez certains représentants de la tribu des Cteniobathynellini. L'aspect général de l'appendice et l'absence de phanères sur la région pénienne rappellent le pénis de Cteniobathynella leleupi (Delamare et Chappuis) (Delamare Deboutteville et Serban, 1974), le développement accusé de l'exopodite se retrouve chez Ctenophallonella mutlumuviensis Coineau et Serban, la réduction totale de l'endopodite (article et poils) est également propre au pénis de Haplophallonella heterodonta Serban et Coineau, tandis que la présence d'une région pénienne proéminente et dépassant l'extrémité distale du basipodite caractérise aussi le péréiopode VIII mâle de Habrobathynella milloti (Delamare et Paulian) (Delamare Deboutteville et Serban, 1974a); ajoutons à ces observations le fait que, dans la tribu des Cteniobathynellini, l'exopodite se trouve toujours sur la face distale du basis. En même temps, le pénis de l'espèce en question s'individualise par deux caractères qui, jusqu'à maintenant, n'ont pas été mis en évidence chez les Cteniobathynellini: la région pénienne est formée d'un grand lobe portant un lobule; le basipodite est complètement englobé dans la région basale de l'appendice, sa présence étant indiquée seulement par le long poil postérieur. Le premier caractère marque une extrême simplification de la région pénienne; le second montre une concentration maximale de la structure pénienne 
par la fusion de la face médiale du basis avèc la paroi de la région pénienne, le bord postérieur de cette dernière s'arrêtant au niveau de la limite distale du basis (fig. 5 D).

\section{Les genres Acanthobathynella Coineau et Lamtobathynella n.g.}

La comparaison de l'ensemble des caractères d'A. knoepffleri et Lt. pentodonta montre une forte ressemblance quant à la morphologie générale de la plupart des appendices et au nombre et la disposition des phanères. A l'exception de la morphologie particulière de tous les phanères chez A. knoepffleri, les différences les plus marquées des deux taxa se manifestent au niveau de la chétotaxie des antennules, de la morphologie de la partie masticatrice mandibulaire et des péréiopodes VIII mâles, différences que nous analysons ci-dessous.

Chez Acanthobathynella, les antennules ne portent pas des bâtonnets hyalins, tandis que les poils de type a et les poils en lancette son remplacés par les poils flagellés, d'une part et les poils tronqués de l'autre (Coineau et Serban, 1974).

La mandibule d'Acanthobathynella, ayant une partie incisive peu développée, ne porte ni la griffe qui se trouve à la base de la partie mentionnée ni les deux suivantes; dans la région où chez Lt. pentodonta se trouve le lobule s'individualise un lobe de grande taille, muni d'une petite griffe et deux protubérances.

L'étude des péréiopodes VIII mâles de Lt. pentodonta nous permet d'éliminer quelques erreurs de la description de ces appendices chez $A$. knoepffleri (fig. 7). En effet, le prolongement apical du basipodite, que nous avons considéré comme étant l'endopodite (Coineau et Serban, 1974), est l'exopodite, tandis que la formation attribuée à l'exopodite ne représente que le lobe externe; ce dernier est fusionné avec la région basipodiale.

Nous donnons ci-dessous la diagnose modifiée du genre Acanthobathynella Coineau:

Chétotaxie générale comportant des phanères courts et robustes. Péréiopode VIII mâle: basipodite complètement englobé dans la région basale de l'appendice (région basipodiale); région pénienne formée d'un seul lobe muni d'une épine; lobe externe fusionné avec la région basipodiale; exopodite, incomplètement individualisé, situé dans la partie distale de la région basipodiale; endopodite absent. Péréiopodes VIII femelle très petit, glabre. Métastome glabre. Mandibule à partie incisive portant 3 dents; un grand lobe est individualisé dans la région caudale et ventrale du corps mandibulaire; il porte une petite griffe et 2 protubérances. Antennule de 6 articles, à chétotaxie formée de deux types particuliers de poils. Antenne pentaarticulée. Maxillule à endite proximal portant 4 phanères; article distal muni 
de 5 phanères sur le bord interne et 2 sur le bord externe. Maxille, biarticulée, avec l'article proximal glabre. Furca à. 5 phanères.

\section{RESUME}

A la suite de la description de Lamtobathynella pentodonta n. g. n. sp., nous envisageons les relations du nouveau genre avec les genres Leptobathynella et Parvulobathynella et nous donnons des remarques au sujet des traits les plus caractéristiques des péréiopodes VIII mâles. Ce travail se termine par une discussion à propos de différences entre Lamtobathynella pentodonta. et Acanthobathynella knoepffleri Coineau.

\section{BIBLIOGRAPHIE}

COINEAU, N. 1967. Acanthobathynella, Nouveau genre de Syncaride africain (Côte d'Ivoire). C.R. Acad. Sc. Paris, Sér. D. 256: 1988-1990.

COINEAU, N. et E. SERBAN, 1974. Le genre Acanthobathynella Coineau (Podophallocarida, Bathynellacea) et la sous-famille des Acanthobathynellinae nov. Ann. Spéléol., 28 (3): 503-516.

DELAMARE DEBOUTTEVILLE, Cl. et E. SERBAN. Sur les péréiopodes VIII mâles de Cteniobathynella leleupi (Delamare et Chappuis) (Parabathynellidae, Bathynellacea). Ann. Spéléol., 29 (3. 1): 373-379.

DELAMARE DEBOUTTEVILLE, $\mathrm{Cl}$. et SERBAN 1974a. Contribution à la connaissance des péréiopodes VIII mâles de Habrobathynella milloti (Delamare et Paulian) (Parabathynellidae, Bathynellacea). Ann. Spéléol. 29 (3): 381-387.

DELAMARE DEBOUTTEVILLE, $\mathrm{Cl}$. et E. SERBAN, 1979. Odontobathynella amazonica n.g., n.sp., Leptobathynellidé nouveau du Brésil (Malacostraca Bathynellacea). Bull. Soc. Sci. nt. Tunisie, 14:5-20.

NOODT, W. 1963. Subterrane Crustacean der Zentralen Neotropis. Zur Frage mariner Relikte im Bereich des Rio Paraguay-Parana-Amazonas-Systems. Zool. Anz. 171 (1-4): 114-147.

NOODT, W. 1965. Natürliches System und Biogeographie der Syncarida (Crustacea Malacostraca). Gewässer und Abwässer, 37-38: 77-186.

NOODT, W. 1972. Brasilianische Grundwasser-Crustacea, 2. Nannobathynella, Leptobathynella und Parabathynella aus der Serra do Mar von Sao Paulo (Malacostraca, Syncarida). Crustaceana, 23:152-164.

SCHMINKE, H.K. 1972. Evolution und Homologisierung des Mandibel-type der Bathynellacea (Crustacea, Malacostraca). Z.f. zool. Systematik u. Evolutionsforschung, 10 (3): 174-180.

SCHMINKE, H.K. 1973. Evolution, System und Verbreitungsgeschichte der Familie Parabathynellidae (Bathynellacea, Malacostraca). Mikrofauna Meeresboden, 24:1-192.

SCHMINKE, H.K. 1981. Adaptation of Bathynellacea (Crustacea, Syncarida) to Life in the Interstitial ("Zoea Theory»). Int. Revue. ges. Hydrobiol, 66 (4): $575-637$.

SERBAN, E. 1980. La mandibule et l'individualisation des ensembles évolutifs majeurs dans l'ordre des Bathynellacea (Malacostraca, PodophalloCarida). Bijdragen tot de Dierkunde, 50 (1):155-189.

SERBAN, E. et N. COINEAU 1975. Haplophallonella heterodonta, n. g., n. sp., Parabathynellidé (Podophallocarida, Bathynellacea) d'Afrique (Côte d'Ivoire). Trav. Inst. Spéol. "Emile Racovitza", 14:51-70. 